bility or activity of a given protein. Alternative or more efficient methods for fractionating and purifying the proteins are clearly detailed and many of them are useful to someone struggling with an expression system or purification scheme of their own. It is refreshing to see the resurgence of interest in the practical and theoretical aspects of protein production and purification.

It is often forgotten that much of the original impetus for developing recombinant cloning technologies was to overcome the limiting reagents problem. Many of the proteins such as repressors, activators, hormones and processing enzymes that were of paramount importance to investigators in the early 1970 s were quite rare and in extremely limited supply. It was mandatory to clone and overexpress the targets in order to study them. But since it became much easier (and in some people's opinion, more fun) to clone, sequence and characterize a gene than to analyse the biophysical nature of the protein encoded by the gene, many of the original goals were superseded. It is therefore pleasing to see individuals on the editorial board who love the methodology-oriented practice of protein purification itself. Robert Scopes, one such member, stated in the preface to his book Protein Puri-

\section{Electrifying separations}

\section{David C. Schwartz}

Applied and Theoretical Electro-
phoresis. Editor-in-chief Carl. R. Merril.
Macmillan. $6 / y r . \pm 80, \$ 140$.

SINCE the time of Tiselius, electrophoresis has attracted an interesting assortment of scientists united in their quest for either putting their favourite purified molecules into bottles or understanding the physical mechanisms behind the electrophoretic processes responsible for these separations, or possibly both. As can be expected, electrophoresis research is very interdisciplinary with its breadth covering physicists' modelling systems on Cray supercomputers, to clinicians examining spinal fluid for disease. It is not surprising that Linus Pauling helped launch molecular biology, in part, with his remarkable electrophoretic analysis of globin mutations responsible for sickle-cell anaemia more than four decades ago. Since then, electrophoresis-based analsysis has played a pivotal role in the biological sciences. It is this blend of physics and biology that makes Applied and Theoretical Electrophoresis a valuable journal to physically oriented scientists searching for fication: Principles and Practice, "The fascination of isolating a reasonably pure enzyme from a complex natural soup of protein remains with me; as a challenge and an academic exercise I still spend much time on enzyme purification even when I have no real use for the final product!"

In today's world of new start-up ventures in everything from journals to biotechnology companies, one must take a cautious wait-and-see attitude before investing one's precious manuscript or life's savings. The future seems bright for these two journals, but the competition for quality manuscripts will be stiff. I admonish the members of the editorial boards of the two journals to provide a first-rate theatre for the auditioning of details. Performers, such as the meticulous graduate student or the postdoctoral fellow, should be invited to describe their methods for someone to repeat on the first attempt. The biochemist whose publication list boasts several papers in either of these journals would then be the one to look out for.

Charles S. Craik is in the Departments of Pharmaceutical Chemistry and Biochemistry and of Biophysics, University of California at San Francisco, San Francisco, California 94143-0446, USA.

new physical phenomena to investigate as well as to biologists requiring new methodologies to solve difficult problems in molecular analysis. The journal is, of course, also of great interest to researchers working directly in this field.

Most of the issues have a nice blend of articles covering computer analysis of data, work to characterize and find new separation matrices, as well as the expected array of articles examining a myriad of electrophoresis separation mechanisms. Biochemists and molecular biologists will find that articles are evenly distributed between protein- and nucleic acid-based separations. New electrophoretic techniques are also covered along with numerous broadly useful applications - this will endear the journal to scientists who are not really interested in electrophoresis. Often more specialized electrophoresis journals publish applications with only limited audience appeal. It is also gratifying to see that extensive materials and methods sections accompany most articles, thereby enabling researchers to readily try out new techniques.

Applied and Theoretical Electrophoresis is a journal that should be consumed by most biologists as well as interested physical scientists.

David C. Schwartz is in the Department of Chemistry, New York University, 31 Washington Place, New York, New York 10003, USA.

\section{Going against the stream}

\author{
Barry L. Karger
}

Bioseparation: International Journal of Separation Science in Biotechnology. Editor-in-chief Tony Atkinson. Kluwer. 6/yr. Dfl. 506, \$257, £172 (institutional); Dfl. 285, \$150.50, £97 (personal).

Is this one necessary? Journals seem to increase in number by leaps and bounds, and the field of separation science already has many, including Journal of Chromatography, Preparative Chromatography, LC/GC and Separation Science and Technology. But editors of Bioseparation argue that separation techniques for downstream processing in biotechnology are too spread out among other journals (including those of chemical engineering), and aim to remedy this situation.

After examining the journal I agree that the emphasis on downstream processing is warranted. Furthermore, the quality of the papers is most pleasing. In general, they seem to be very useful, well thought out and well presented. It is fair to say that some of them would be overlooked if they were to appear in a more diverse journal.

Other important aims of the journal are to publish both reviews and issues devoted to special topics. The December 1990 issue dealt with aqueous two-phase separation systems. Such issues have real value in that a great deal of current information on a particular topic can be assembled in one place. This format has also been well incorporated into other journals.

A good start has been made in establishing a high-quality journal for downstream processing in biotechnology. I hope that there will be increased emphasis on high-performance separations in preparative-scale chromatography as well as that placed on preparative-scale electrophoresis. These two topics will clearly evolve as the journal expands its coverage. In general, the journal is recommended as a useful source for downstream processing.

The editorial board consists predominantly of engineers rather than chemists and, therefore, large-scale separations feature greatly in the journal. Will the editors also become interested in process analysis?

Barry L. Karger is the Director of the Barnett Institute of Chemical Analysis and Materials Science, Northeastern University, 360 Huntington Avenue, Boston, Masachusetts 02115, USA. 